\title{
The effect of particulate matter on paper degradation
}

\author{
Josep Grau-Bové ${ }^{*}$, Bojan Budič², Irena Kralj Cigić $^{3}$, David Thickett ${ }^{4}$, Stefania Signorello ${ }^{5}$ and Matija Strlič ${ }^{1}$
}

\begin{abstract}
Background: In this work we explore the chemical effects of particulate matter on paper. We exposed paper made of pure cellulose to the environment in different locations in central London, outdoors (in sheltered conditions) and indoors, for a period of up to 6 months. We monitored particulate matter (PM) deposition by counting the particles deposited every month with a scanning electron microscope. We analysed elemental composition of the deposited particles using inductively coupled plasma mass spectrometry. After accelerated degradation of the exposed samples, we determined the degree of polymerisation using viscometry.

Results: We observed higher deposition rates and higher metal concentration outdoors than indoors. Elemental analysis of the deposited particles revealed the presence of some transition metals ( $\mathrm{Fe}, \mathrm{Cu}, \mathrm{Cr}$ ) that can contribute to the degradation of cellulose fibres through the Fenton reaction. By comparing the degree of polymerisation of protected, unprotected and unexposed samples we could determine the relative contribution of PM deposition on the increase of the degradation rate. We found that the surface concentration of iron correlates with the reduction in the degree of polymerisation of the exposed paper.
\end{abstract}

Conclusions: The results suggest that the presence of Fenton metals in PM has a significant effect on the acceleration of the degradation of cellulose. However, we estimate that this will unlikely occur at the levels of area coverage by PM that are typically avoided in indoor heritage through preventive maintenance and cleaning.

Keywords: Paper, PM, Indoors, Exposure

\section{Background}

It is well known that the deposition of particulate matter (PM) on outdoor surfaces can accelerate material degradation. For example, the presence of diesel particulate matter has been related to the decay of carbonaceous [1] and silicate stones [2]. Several corrosion products of copper have been identified on statues where soot was also present [3]. However, there is an important lack of literature about the degradative effects of particulate matter deposition on the surface of paper, leather, textiles, paintings, varnishes and any other organic material typically found indoors.

Quite differently, the main concern of studies of particulate matter deposition indoors seems to be area

\footnotetext{
*Correspondence: josep.grau.bove@ucl.ac.uk

${ }^{1}$ Institute for Sustainable Heritage, University College London, Gower

Street, London, UK

Full list of author information is available at the end of the article
}

coverage and its aesthetic consequences. This concern is justified: visitors can perceive deposited particles at very low levels of area coverage. Studies on the ability of the human eye to detect soot deposition show that some observers are able to discern a soiled surface from a clean one when the covered area is between 1 and $3.5 \%$ depending on surface properties [4], or when coverage is as low as $0.2 \%$ if the particles are very large $(0.5-1 \mathrm{~mm})$ [5]. Values between 3 and $6 \%$ are normally taken as thresholds of unacceptable deposition, for example, Historic Royal Palaces (London, UK) aim to reduce deposition to $3 \%$ monthly area coverage [6].

A question which remains unresolved is to determine how do these values of area coverage relate with the capacity of the particles to chemically interact with the substrate. The combinations of possible PM compositions and substrate types are infinite, but in order to simplify our question, let us concentrate on indoor organic materials, which could be argued to be the most vulnerable to

\section{Springer}

(c) 2016 Grau-Bové et al. This article is distributed under the terms of the Creative Commons Attribution 4.0 International License (http://creativecommons.org/licenses/by/4.0/), which permits unrestricted use, distribution, and reproduction in any medium, provided you give appropriate credit to the original author(s) and the source, provide a link to the Creative Commons license, and indicate if changes were made. The Creative Commons Public Domain Dedication waiver (http://creativecommons.org/ publicdomain/zero/1.0/) applies to the data made available in this article, unless otherwise stated. 
particulate matter. A double question arises: Does particulate matter have a chemical effect on organic materials? If so, is this chemical effect significant at the levels of area coverage that are commonly used as thresholds of acceptable deposition?

The scarce evidence available in the literature suggests that this degradation may occur. It has been shown that the presence of fine particulate matter $\left(P M_{1}\right)$ on the surface of paper is related with an increase of its degradation rate [7]. Camuffo hypothesised [8] several possible degradation pathways that are related to typical components of PM: (i) S-rich material can cause discolouration of paintings. (ii) Ammonium sulfate can induce bloom on varnish. (iii) The presence of $\mathrm{CaSO}_{4}$ favours the adsorption of soot (iv) and Fe-rich particles can catalyse oxidative processes.

We consider that the fourth process, the presence of iron and other transition metals, should be further investigated. More specifically, iron is one of several metals that can catalyse the degradation of cellulose through the Fenton reaction [9]. This is the name given to the generation of a hydroxyl radical from hydrogen peroxide in the presence of a suitable transition metal, a reaction discovered by Henry Fenton in 1894 [10]:

$$
\mathrm{Fe}^{2+}+\mathrm{H}_{2} \mathrm{O}_{2} \rightarrow \mathrm{Fe}^{3+}+\mathrm{OH}^{\cdot}+\mathrm{OH}^{-}
$$

We suspected that, through this mechanism, particles embedded in paper could accelerate the degradation of the surrounding cellulose fibres. To investigate this issue, we studied the chemical degradation of paper samples exposed to environmental particulate matter. We specifically focused on chemically pure cellulosic fibres as these could be seen as the cellulosic material most susceptible to degradation. As such, the effects of particulate matter observed in this article could be seen as intentional exaggeration where in real materials the effect is likely going to be less pronounced.

\section{Methods}

We exposed samples of Whatman filter paper No. 1 (Maidstone) to the environment in outdoor and indoor locations, counted the amount of deposited particles and assessed the degradation by measuring the degree of polymerisation (DP). Whatman paper sample type provides a very simple model system. This sample type has many advantages: firstly, it is non-sized and contains no filters or additives, which makes it a good reference sample [11]. Furthermore, it is a well-known type of paper, which allows the data to be used and compared with previous research. It has been used previously to approximate the effect of the environment on organic materials [12]. Since the sample $\mathrm{pH}$ is close to neutral, it is likely susceptible to both acid-catalysed cellulose degradation and oxidation, depending on the composition of the deposited particles. It has the disadvantage of being relatively thick, which contributes to the dilution of the effects, but we considered that other alternatives would add complexity to the experimental design.

Clearly, the effect of particulate matter on paper must be very localised, because it is likely that particles can only interact with the immediately adjacent fibres.The thickness of cellulose fibre threads of our samples is generally between 5 and $20 \mu \mathrm{m}$, which is comparable to the diameter of particulate matter. Figure 1 shows some particles embedded in a piece of Whatman filter paper, consisting of pure cellulosic fibres, heavily soiled with environmental PM collected outdoors. Figure 1 corresponds with Month 5 of exposure of the Window sample. This SEM image conveys a sense of the relative size of the particles and the cellulosic fibres.

This experiment faced two main challenges. Firstly, we expected degradation to be limited to the surroundings of the particles. However, DP measurements are a bulk assessment of the degradation of cellulose, with spatial resolution about 1000 times the diameter of a typical particle, which means that we can only obtain information on the average properties of a paper sample. We measured degradation which was probably local and disperse, using bulk analysis of the paper and yet, as we shall see, we were able to show a statistically significant effect of particles on the overall degradation. The second major challenge of this experiment was to isolate the effect of PM on paper samples that had been exposed to the urban environment, and had therefore been in contact with pollutant gases and fluctuating $T$ and $R H$ conditions.

\section{Paper exposure to environmental PM}

We exposed racks of samples of Whatman paper alongside carbon stickers for particle counting in five different locations around London, two indoors and three outdoors, for a period of 6 months (20/2/2013 to 20/7/2013). The outdoor samples were in sheltered locations. Four of these racks were in Apsley House, located near a busy roundabout in Hyde Park Corner, while one was in the Wellcome Collection building, in Euston Road, both in central London. These are two of the most polluted roads in London, where the average concentration of PM2.5 during the monitoring period was around $16.7 \pm 11.5 \mu \mathrm{g} / \mathrm{m}^{3}$, with a maximum of $80 \mu \mathrm{g} / \mathrm{m}^{3}$. The exposure locations were:

1. Plate and China Room, Apsley House, ground floor. Top of a display case.

2. First Floor, Apsley House, first floor. Top of a display case. 


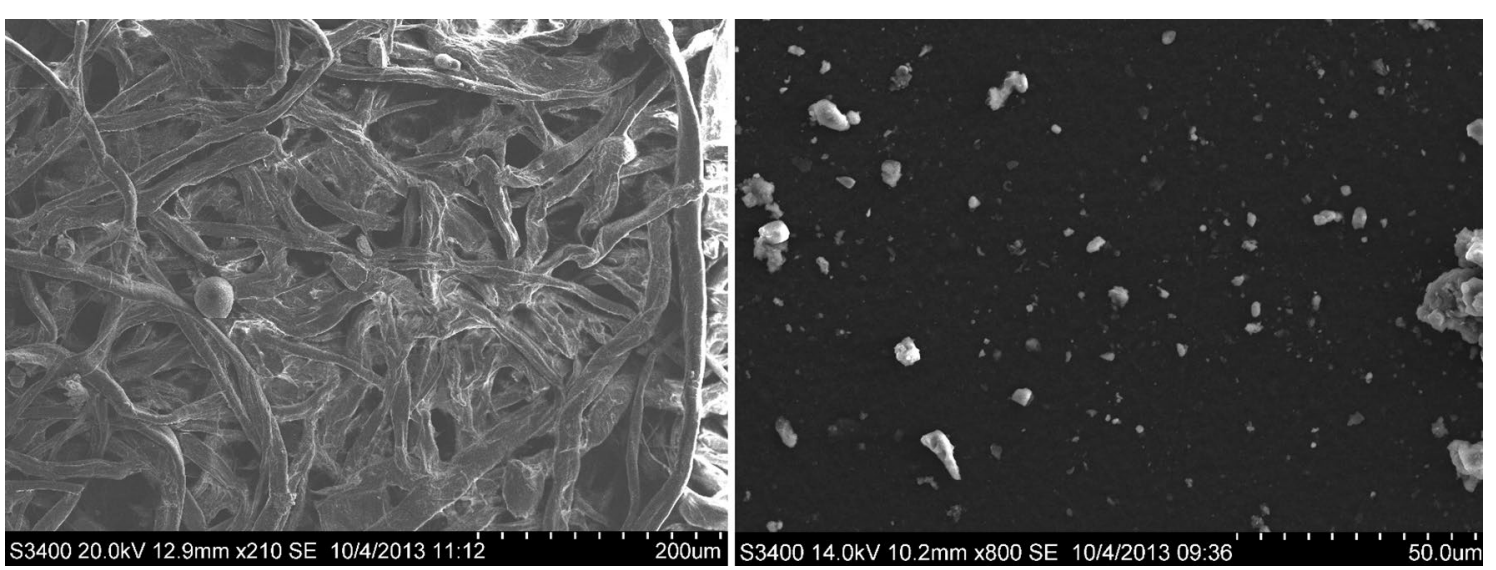

Fig. 1 Paper sample. Micrographs of PM embedded in a sample of Whatman paper and the carbon stub used to collect particles in the same location. The cellulosic fibres are of a size comparable to the diameter of the deposited particles. The image corresponds to the Window location, in Month 5 of the exposure period

3. Service Stairs, Apsley House, basement, open to outdoor environment. Protected under a roof, $19 \mathrm{~m}$. from the nearest road.

4. Window, Apsley House, below street level. Sheltered in a window recess, $19 \mathrm{~m}$. from the nearest road.

5. Wellcome Collection building, roof. Protected from rain and wind in a well-ventilated shed.

We exposed the samples for 1-6 months, removing a sample from each location every month. Our motivation to expose samples outdoors and indoors was to cover a wide range of deposition rates, and to ensure we had a high proportion of fine particulates and particles with different chemical composition. Outdoors, we expected to find road debris and combustion particles, which contribute with organic and inorganic carbon and metallic traces $[13,14]$. Particulate matter inside Apsley House is mostly of outdoor origin, and therefore we expected to find a lower amount of the same chemical species. Additionally, some of the indoor particles, particularly the coarser, consist of dust carried by visitors, which may have a different composition.

The paper samples measured 3 by $12 \mathrm{~cm}$ and were laid out in a sample rack that contained two paper samples and a SEM stub for particle counting for every month of exposure (a diagram of the sample rack is provided in Additional file 1: Annex 1). In order to isolate the effects of particulate matter and gaseous pollutants, we covered half of the samples with a permeable polymer membrane (Clopay MicroPro). This membrane protected the sample from particulate matter and light, but allowed gases to penetrate. We used a UV Logger (Hanwell ULM-Universal Light Meter) to measure that it blocked $99.1 \pm 0.2 \%$ of the incident UV light, and we used diffusion tubes
(Gradko) to ascertain that the membrane allowed $77 \%$ of the $\mathrm{O}_{3}$ and $94 \%$ of the $\mathrm{NO}_{2}$ present in the environment to reach paper samples, which is enough to ensure that the paper interacted with these gases. On the other hand, SEM analysis of the protected paper samples did not reveal the presence of a measurable amount of particulate matter larger than $0.05 \mu \mathrm{m}$.

The total particle counts per unit area in each monitoring location can be seen in Fig. 2. We monitored particulate matter deposition by counting the particles deposited every month with an SEM microscope (Hitachi S-3400N). We produced between 10 and 15 screen captures of the sample in a grid, with a magnification of $\times 500$. We counted all particles with diameters above $0.5 \mu \mathrm{m}$, and used this data to calculate deposition rates, using the time of exposure and the dimensions of the analysed area. If deposition was very low and inhomogeneous, and thus particles were not homogeneously distributed in all the SEM micrographs, we considered the sample to be below the detection threshold. In two of the locations (outdoors and indoors in Apsley House), we also monitored the concentration of suspended particulate matter in different size modes $(0.5-1,1-2.5,2.5-5$ and $5-20 \mu \mathrm{m})$ every minute using three laser particle counters (Dylos DC1010).

Not all the exposed paper samples could be used in the final experiments. Either a problem with the exposed paper or a problem with the SEM stubs for particle counting resulted in loss of an experimental point. Firstly, we had to discard 6 out of 30 samples due to problems related to particle counting. Some of these were discarded merely because in the initial months, the deposition on some indoor samples was below the detection threshold. Some samples were also accidentally damaged during collection 

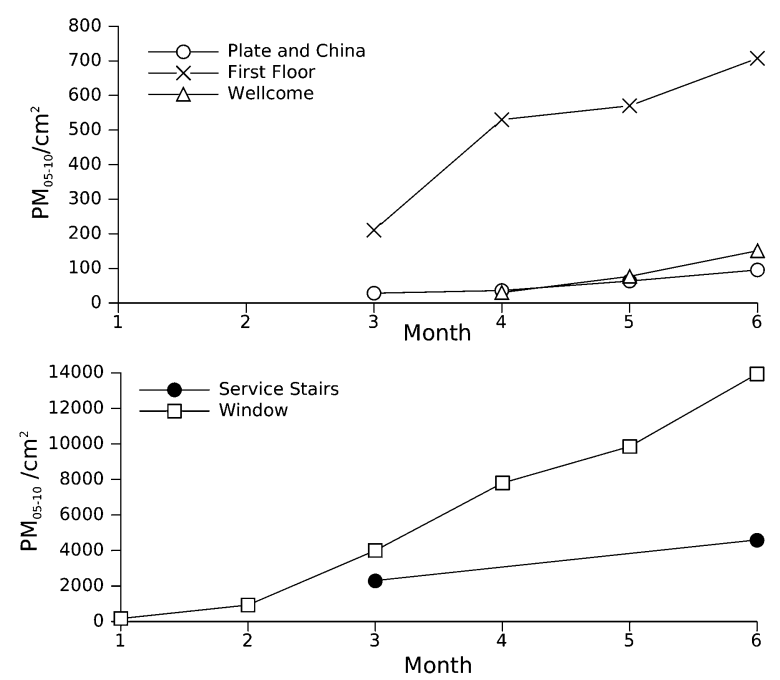

Fig. 2 Deposition measurements. Accumulation of particles per unit area in the monitoring locations

or transport, causing the loss of an important amount of deposited particles. We also experienced some issues related with the exposed paper. For example, some outdoor samples were accidentally damaged by water, showing visible staining. In total we lost 9 paper samples and 6 carbon stubs for particle counting (these issues are summarized in Additional file 2: Annex 2).

\section{Chemical analysis}

We analysed the elemental composition of the deposited particles using inductively coupled plasma mass spectrometry (ICP-MS). Prior to the analysis the filter samples were digested in a microwave oven (Milestone, Ethos 1). Firstly, samples were weighted directly into polytetrafluoroethylene (PTFE) vessels, to which $8 \mathrm{ml}$ of concentrated $\mathrm{HNO}_{3}$ (s.p. quality, Merk Darmstadt) and $2 \mathrm{ml}$ of $\mathrm{H}_{2} \mathrm{O}_{2}$ (Merk Darmstadt) were added. The heating program for digestion consisted of a ramp of $20 \mathrm{~min}$ until $180{ }^{\circ} \mathrm{C}$, followed by a ramp of $10 \mathrm{~min}$ until $220{ }^{\circ} \mathrm{C}$, and ending by holding this temperature for $20 \mathrm{~min}$. Finally, the cooling program lasted $30 \mathrm{~min}$. Once the samples were at room temperature, the vessels were opened, the solutions transfered into $50 \mathrm{ml}$ measurement flasks, the vessels washed with deionized Milli-Q (Merck Millipore) water and measurement flasks diluted with deionised Milli-Q water (purity $18 \mathrm{M} \Omega \mathrm{cm}$ ). 10 sample blanks were also prepared following the same procedure.

The calibration was based on a multielemental calibration solution which contained all the analytes $(\mathrm{Mg}$, Ti, V, Cr, Mn, Fe, Co, Ni, Cu, Zn, As, Sr, Cd, Sb, Tl, Pb) prepared at the following concentrations: 0, 0.01, 0.05, $0.1,0.5,1,5,10,50$ and $100 \mathrm{ngl}^{-1}$. $\mathrm{HNO}_{3}$ was added to the calibration solutions to the same concentration found in the sample solutions. The multielemental solution was prepared from certified multielemental and single element solutions (Merc, Darmstadt, Germany). The analyte concentrations were measured by ICP MS under experimental conditions, presented in Additional file 3: Annex 3. Finally, the average blank value was subtracted from the analyte concentration.

ICP-MS can detect very small traces of metals and is capable of quantitatively measuring analytes down to a few nanograms [15]. This allowed us to obtain accurate measurements of the surface concentration of metals using only $1 \mathrm{~cm}^{2}$ of material from each sample, which is a relatively small amount compared to the total area available (each paper sample measured approximately $35 \mathrm{~cm}^{2}$ ). It was necessary to minimise the amount of sample used for chemical analysis in order to have enough paper available to carry out viscometric analysis. Therefore, we were able to remove $1 \mathrm{~cm}^{2}$ of paper for months 2,4 , and 6 and from four different locations. The results of the chemical analysis are summarised in Table 1.

\section{Viscometric determination of DP}

With the purpose of accelerating the effects of the deposited particles on the paper substrate, we additionally aged half of each sample (protected and not protected) for 3 weeks at $80^{\circ} \mathrm{C}$ and $65 \%$ RH (ISO 5630-3). The half of each sample that was not aged was used to the determine the effect of the ageing process. We then used viscometry to measure the change in the intrinsic viscosity of cellulose, $\eta$, from which we calculated the degree of polymerisation, DP, which is proportional to the decrease in the average molecular weight of cellulose. DP was calculated on the basis of the Mark-Howink-Sakurada equation [16]:

$$
D P^{0.85}=1.1 \eta
$$

We determined the intrinsic viscosity, $\eta$ following ISO 351:2010. This standard describes the experimental procedure to determine the viscosity using a capillary viscometer. We used three analytes from every paper sample, and we obtained a satisfactory repeatibility (with a coefficient of variation between analytes close to $1 \%$ in the determination of DP). However, note that the main source of uncertainty in our experimental procedure is not the viscometric determination of DP, which is highly repeatable for any given sample, but the variation between samples due to uncontrollable environmental variation and particulate matter composition. 
Table 1 Elemental composition (measured in $\mu \mathrm{g} \mathrm{cm}^{-2}$ ) of the samples of particulate matter at the end of the experimental period

\begin{tabular}{lcccc}
\hline & Plate and China & Window & Service Stairs & Wellcome \\
\hline $\mathrm{Mg}$ & 0.70 & 7.23 & 3.18 & 0.88 \\
$\mathrm{Ti}$ & 0.06 & 0.66 & 0.08 & 0.06 \\
$\mathrm{~V}$ & 0.001 & 0.041 & 0.003 & 0.002 \\
$\mathrm{Cr}^{\mathrm{a}}$ & 0.01 & 0.10 & 0.03 & 0.02 \\
$\mathrm{Mn}$ & 0.01 & 0.42 & 0.13 & 0.02 \\
$\mathrm{Fe}$ & 0.6 & 22.9 & 1.6 & 1.1 \\
$\mathrm{Co}$ & $<0.001$ & 0.021 & 0.002 & $<0.001$ \\
$\mathrm{Ni}$ & 0.02 & 0.05 & 0.04 & 0.03 \\
$\mathrm{Cu}$ & 0.05 & 0.61 & 0.12 & 0.06 \\
$\mathrm{Zn}$ & 0.15 & 4.58 & 6.31 & 0.16 \\
$\mathrm{As}$ & 0.11 & 0.07 & 0.73 & 0.01 \\
$\mathrm{Sr}$ & 0.01 & 0.30 & 0.10 & 0.01 \\
$\mathrm{Cd}$ & $<0.01$ & 0.01 & $<0.01$ & $<0.01$ \\
$\mathrm{Sb}$ & $<0.01$ & 0.07 & 0.01 & $<0.01$ \\
$\mathrm{Tl}$ & $<0.01$ & $<0.01$ & $<0.01$ & $<0.01$ \\
$\mathrm{~Pb}$ & 0.01 & 0.99 & 0.09 & 0.02 \\
\hline $\mathrm{Th}$ & & 0.01 & & \\
\hline
\end{tabular}

${ }^{a}$ The elements that can contribute to the Fenton reaction

After accelerated degradation and DP determination with viscometry, we calculated the loss of DP due to PM deposition as the difference between the DP of the unprotected samples (which were affected by PM and other factors such as gases) and the protected samples (which were affected by all environmental factors except PM):

$$
D P_{\text {loss }}=D P_{\text {unprotected, aged }}-D P_{\text {protected, aged }}
$$

The parameter $D P_{\text {loss }}$ indicates how much paper has degraded due to the presence of particles in comparison with a baseline value of degradation caused by all factors except particles (mainly gaseous pollutants). However, its value depends on the initial state of degradation before aging. In order to know how much do particles accelerate degradation, we defined the relative degradation, $x$, as:

$$
x=\frac{D P_{\text {unprotected }, \text { not aged }}-D P_{\text {unprotected }, \text { aged }}}{D P_{\text {protected }, \text { not aged }}-D P_{\text {protected }, \text { aged }}}
$$

$x$ could be interpreted as the relative increase in degradation due to PM deposition over the baseline of degradation that could be expected from the rest of the environment. In other words, a sample with deposited particles experiences a reduction of DP after ageing $x$ times higher than a sample without particles. The disadvantage of the relative degradation, as we shall see, is that it requires the operation with measurements of DP subject to some experimental error, and therefore the error associated with the results increases due to error propagation. It provides, however, an interesting measure that allows an assessment of the relative importance of the PM-induced degradation.

\section{Results and discussion}

Figure 3 shows the relationship between $D P_{\text {loss }}$ with the total particle count $\left(P M_{0.5-10}\right)$, indicating the sample location and the collection month. This figure includes data from all locations and times of exposure for which a particle count could be obtained. A logarithmic horizontal axis is needed due to the large dispersion on the number of deposited particles (from 10 to 10,000 particles per $\mathrm{cm}^{2}$ ). We can see that there is a weak overall correlation, however, the degradation of paper in some locations, namely the Plate and China Room (indoors) and the Window (outdoors) exhibits a clear gradual increase with particle number. This relationship is not visible in the First Floor and Wellcome locations.

Figure 3 also reveals that very different numbers of particles in different locations result in relatively similar levels of DP loss. This is particularly evident, for example, when comparing Month 1 of the Window sample with Month 3 of the Service Stairs sample. Both experimented a decrease of DP of approximately 200, but the number of particles needed to reach this level of degradation is more than ten times higher in the Service Stairs location, and the time of exposure is 2 months longer. A possible explanation could be that particles from different locations have different effects on $D P_{\text {loss. }}$. We suspect that the different degradative effects of PM on paper might be related to varying chemical composition. However, this cannot be proved with the evidence displayed in Fig. 3 which only indicates that, while there is a poor correlation between particle number and $D P_{\text {loss }}$, this relationship seems to be clear in specific locations. To investigate this issue, we explored the relationship between degradation and PM composition.

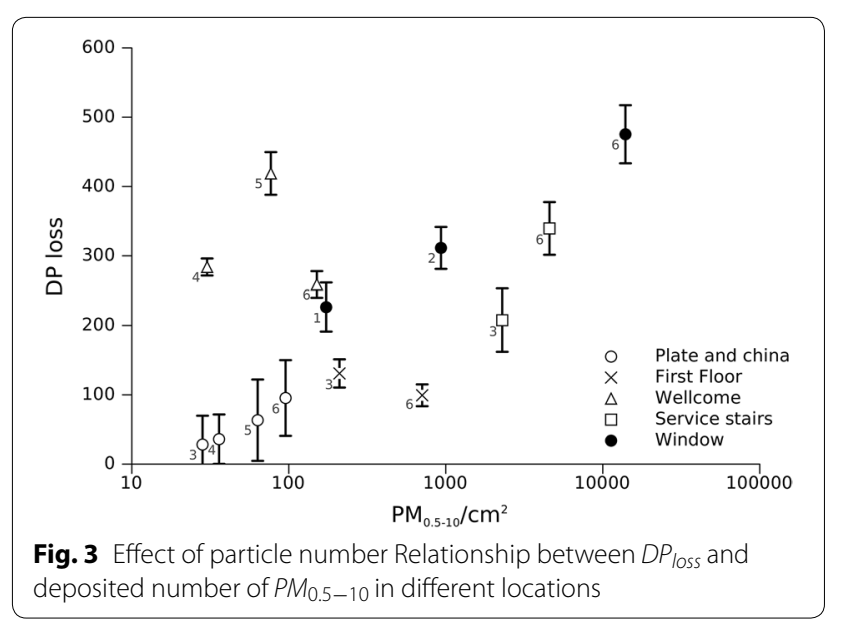


The results of elemental composition of the PM deposits shown in Table 1 are comparable to other elemental analyses of urban $P M_{10}$ in the UK. In Birmingham, for example, it was also found that the most abundant metal (in mass) was Fe (38 \%) followed by $\mathrm{Mg}$ (29\%), Ca (14\%), Zn (5.6 \%) and others [17]. Iron concentrations are higher in street sites than in rural sites, and can indicate a contribution of suspended road dust [18]. We have detected iron in all our sampling locations, indoors and outdoors. Road dust may explain the origin of iron in the outdoor locations of Apsley House, which were located below the street level in a busy road. Iron has also been found to be the second most abundant metal in Diesel Particulate Matter (DPM) after $\mathrm{Ca}$, followed by $\mathrm{Mg}$, and $\mathrm{Cr}, \mathrm{Zn}$ [19]. Since this analysis includes all particles without size discrimination the results probably show a combination of both sources of Fe: coarse road dust and fine DPM. Table 1 shows that some elements, such as $\mathrm{Fe}, \mathrm{Mg}, \mathrm{Mn}$ or $\mathrm{Ti}$, are more abundant outdoors than indoors by an order of magnitude. $\mathrm{Ti}$ is also a product of the combustion of fossil fuels [20], which explains its relatively higher presence outdoors. We also observed that there is no correlation between the amount of iron and the number of particles, which indicates that the particles collected in each location have different origins and different iron concentrations.

Several of the detected metal ions can contribute to the Fenton reaction: $\mathrm{Fe}, \mathrm{Cu}$ and $\mathrm{Cr}$. Given that iron is the most abundant of these, we will use it for the following analysis. Some of the iron that can be expected in our monitoring locations, such as iron originated in car brakes, is originally in metallic form, which cannot catalyse the Fenton reaction. However, iron in particulates oxidizes quickly after emission, as has been observed for particles emitted from car [21] and train [22] brakes. The higher abundance of iron outdoors might explain the higher $D P_{\text {loss }}$ observed in outdoor samples such as the Apsley House window. In fact, when we plot the $D P_{\text {loss }}$ against the iron concentration on the paper surface, Fig. 4, we obtain a much clearer correlation. Once more, the horizontal axis needs to be logarithmic in order to visualize the differences between the smaller concentrations, given the wide range of values of Iron concentration. The reader will note that there are more experimental points in this plot than in Fig. 3 because the measurements of Fe concentration are unaffected by the loss of SEM stubs for particle counting (see Additional file 2: Annex 2). Note also that some experimental points in this correlation correspond to interpolated concentrations of iron. This is because elemental analysis with ICPMS was only available for Months 2, 4, and 6. However, considering that in all the locations this composition increases approximately linearly, we found it justified to interpolate, using linear interpolation, the concentrations

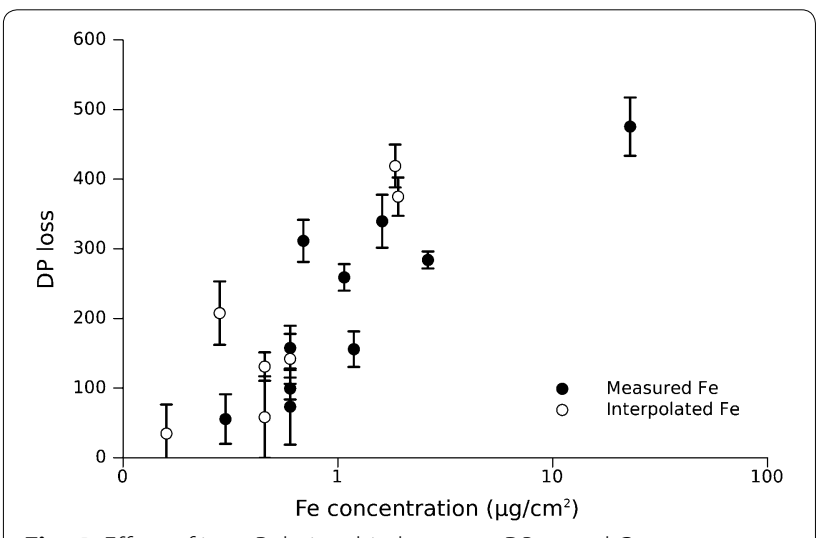

Fig. 4 Effect of iron. Relationship between $D P_{\text {loss }}$ and $C_{F e}$

of Months 1, 3 and 5 (interpolation could lead to apparent linearity, for this reason the interpolated points are clearly marked in Fig. 4).

Figures 3 and 4 show that there is a relationship between $D P_{\text {loss }}$, the number of deposited particles and the iron content of the particles. In order to quantify this relationship we produced a multiple linear regression relating $D P_{\text {loss }}$ with both the number of particles on the surface $(N)$ and the iron concentration $C_{\mathrm{Fe}}$. The resulting regression reads: $D P_{\text {loss }}=\ln (N) \times(36.24 \pm 3.86)+\ln (\mathrm{Fe}) \times(81.63 \pm 31.71)$ with $R^{2}=0.96$ and $\mathrm{f}$ value $=24.6$. The factor multiplying the iron concentration is larger than the factor multiplying $N$, indicating that the composition of particles explains the observed degradation better than the amount of particles. Of course this is only valid if $C_{\mathrm{Fe}}$ and $N$ cover the same range of $D P_{\text {loss }}$.

Clearly the effects of particles are not equal in the different locations. Figure 5 shows the mean value of the relative increase in degradation, $x$ (Eq. 4) for each sample at the end of the monitoring period (Month 6). We can readily see that in the indoor samples (Plate and China, First Floor) the contribution of particles to the overall degradation is not statistically significant ( $x$ is close to 1 ). The contribution of PM to degradation is significant in the Service Stairs and, particularly, in the Window sample. As expected, the relative degradation, $x$, is higher in the locations where the PM deposits contain a higher concentration of Fe. These results indicate that, depending on their composition, particulate matter can increase the degradation of cellulose between approximately 1.1 and 1.5 times. We consider that this level of degradation is not high, according to the following criteria: The most degraded sample, the Window sample, received a deposition that corresponds to $4.3 \%$ of area coverage after 6 months of exposure. This value is comparable to the thresholds of deposition usually used by heritage institutions to indicate 


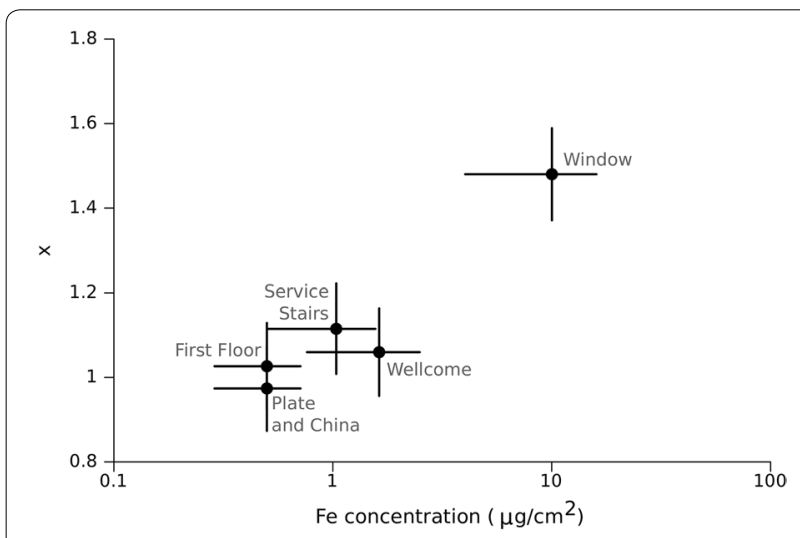

Fig. 5 Iron and relative degradation. Relationship between $C_{F e}$ and the relative contribution of PM to the degradation of paper, $x$. The vertical error bars reflect the uncertainty in the determination of $x$ and the horizontal bars the variation of iron concentration between samples exposed in the same location

the need for cleaning (e.g. $3 \%$ monthly coverage is used by Historic Royal palaces [6]). This indicates that significant damage due to PM deposition will occur on cellulose only when the area coverage exceeds the levels that are generally avoided in indoor heritage environments.

\section{Conclusions}

The fact that PM can accelerate the degradation of cellulose in our experimental conditions is a significant result for two reasons. Firstly, our results show clearly that the effect of deposited PM on paper is not dependent on the amount of particles but, more significantly, on their composition. There is a good indication of a Fe-mediated process, however, this does not preclude any other processes that may take place simultaneously. By protecting some samples from PM with a porous filter, we have isolated the degradative effect of PM from the effect of gaseous pollutants and any other environmental factor. However, there could be other factors related with PM composition affecting the degradation of organic matter, e.g. organic carbon in PM which could oxidize itself and thus lead to undesirable yellowing, as well as any acidity associated with PM. We have not quantified these possible degradation paths as these were insignificant in our case, and remain a matter of future study.

Our experiments have shown that there is a possibility for iron to have a predominant effect, without excluding other factors such as acidity or organic carbon. The observed correlation would be spurious if acidity was the main factor and Fe was correlated with acidity. However, if the Fenton reaction plays a role, our conclusions would be extensible to any comparable organic material, such as textiles, because Fenton reaction-mediated oxidation leads to production of highly reactive organic species such as hydroxyl radicals, reacting with any organic matter at a diffusion-controlled rate.

Secondly, we have shown that, depending on the composition, the presence of PM can increase the degradation rate of paper by a factor between 1.1 and 1.5. Only Sample 5 (exposed in the Window in Apsley House) reached approximately $5 \%$ area coverage, a value which is close to the thresholds of perception usually used in heritage institutions as an indicative level requiring cleaning maintenance. Further experiments, looking in detail at particle size and origin, would be needed to establish a safe value of area coverage that prevents chemical degradation. But as a first approximation, our results suggest that, if organic surfaces are cleaned as soon as deposition becomes visible they are likely to be protected from any significant chemical degradation.

\section{Additional files}

Additional file 1: Annex 1. Diagram of the sample rack used to expose the protected and exposed paper samples.

Additional file 2: Annex 2. Summary of the samples used in the paper degradation experiment.

Additional file 3: Annex 3. Operating parameters of the ICP-MS.

\section{Authors' contributions}

$J G, D T$, and MS designed the experiment and together with SS carried out the monitoring campaign. JG carried out the measurements of particle number and paper degradation and drafted the manuscript. BB and IKC contributed with the chemical analysis and the results section of the paper. All the authors read and approved the final manuscript.

\section{Author details}

${ }^{1}$ Institute for Sustainable Heritage, University College London, Gower Street, London, UK. ${ }^{2}$ Kemijski Institut, Ljubljana, Slovenia. ${ }^{3}$ Faculty of Chemistry and Chemical Technology, University of Ljubljana, Ljubljana, Slovenia. ${ }^{4}$ English Heritage, London, UK. ${ }^{5}$ Wellcome Trust, London, UK.

\section{Acknowledgements}

The authors would like to thank English Heritage and the Wellcome Trust for offering monitoring locations. We also want to thank the staff of the Wellcome Collection and Apsley House for their help during the monitoring campaign.

\section{Competing interests}

The authors declare that they have no competing interests.

Received: 13 May 2015 Accepted: 19 January 2016

Published online: 31 January 2016

\section{References}

1. Rodriguez-Navarro C, Sebastian E. Role of particulate matter from vehicle exhaust on porous building stones (limestone) sulfation. Sci Total Environ. 1996;187(2):79-91. doi:10.1016/0048-9697(96)05124-8.

2. Simão J, Ruiz-Agudo E, Rodriguez-Navarro C. Effects of particulate matter from gasoline and diesel vehicle exhaust emissions on silicate stones sulfation. Atmos Environ. 2006;40(36):6905-17. doi:10.1016/j. atmosenv.2006.06.016.

3. Strandberg H. Reactions of copper patina compounds - i. Influence of some air pollutants. Atmos Environ. 1998;32(20):3511-20. doi:10.1016/ S1352-2310(98)00057-0. 
4. Ligocki MP, Salmon LG, Fall T, Jones MC, Nazaroff WW, Cass GR. Characteristics of airborne particles inside southern California museums. Atmos Environ. 1993;27(5):697-711. doi:10.1016/0960-1686(93)90188-5.

5. Bellan LM, Salmon LG, Cass GR. A Study on the human ability to detect soot deposition onto works of art. Environ Sci Technol. 2000;34(10):194652. doi:10.1021/es990769f.

6. Frame K. Creative conservation risk management: evolving a collection risk management strategy at a major heritage attraction. Collections. 2013;9(1):103-14.

7. Bartl B, Mašková L, Paulusová H, Smolık J, Bartlová L, Vodička P. The effect of dust particles on cellulose degradation. Stud Conserv. 2015. doi:10.117 9/2047058414Y.0000000158.

8. Camuffo D, Brimblecombe P, Van Grieken R, Busse HJ, Sturaro G, Valentino A, Bernardi A, Blades N, Shooter D, De Bock L, Gysels K, Wieser M, Kim O. Indoor air quality at the Correr Museum, Venice, Italy. Sci Total Environ. 1999;236(1-3):135-52

9. Šelih VS, Strlič M, Kolar J, Pihlar B. The role of transition metals in oxidative degradation of cellulose. Polym Degrad Stab. 2007;92(8):1476-81. doi:10.1016/j.polymdegradstab.2007.05.006.

10. Fenton $\mathrm{HJH}$. Oxidation of tartaric acid in presence of iron. J Chem Soc Trans. 1894;65:899-910. doi:10.1039/CT8946500899.

11. Strlic M, Cigic IK, Mozir A, Thickett D, de Bruin G, Kolar J, Cassar M. Test for compatibility with organic heritage materials - a proposed procedure. e-Preserv Sci. 2010;7:78-86.

12. Curran K, Možir A, Underhill M, Gibson LT, Fearn T, Strlič M. Crossinfection effect of polymers of historic and heritage significance on the degradation of a cellulose reference test material. Polym Degrad Stab. 2014;107:294-306. doi:10.1016/j.polymdegradstab.2013.12.019.

13. Mattimaricq M. Chemical characterization of particulate emissions from diesel engines: a review. J Aerosol Sci. 2007;38(11):1079-118. doi:10.1016/j.jaerosci.2007.08.001.

14. Sarvi A, Lyyränen J, Jokiniemi J, Zevenhoven R. Particulate emissions from large-scale medium-speed diesel engines: 2. Chemical composition. Fuel Process Technol. 2011;92(10):2116-22. doi:10.1016/j.fuproc.2011.06.021.
15. Taylor, H.E.: Inductively coupled plasma-mass spectrometry. San Diego: Academic Press; 2001. doi:10.1016/B978-012683865-7/50000-4.

16. Evans R, Wallis AFA. Cellulose molecular weights determined by viscometry. J Appl Polym Sci. 1989;37(8):2331-40. doi:10.1002/ app.1989.070370822.

17. Harrison RM, Tilling R, Romero MSC, Harrad S, Jarvis K. A study of trace metals and polycyclic aromatic hydrocarbons in the roadside environment. Atmos Environ. 2003;37(17):2391-402. doi:10.1016/ S1352-2310(03)00122-5.

18. Janssen NAH, Mansom DFMV, Jagt KVD, Harssema H, Hoek G. Mass concentration and elemental composition of airborne particulate matter at street and background locations. Atmos Environ. 1997;31(8):1185-93. doi:10.1016/S1352-2310(96)00291-9.

19. Sharma M, Agarwal AK, Bharathi KVL. Characterization of exhaust particulates from diesel engine. Atmos Environ. 2005;39(17):3023-8. doi:10.1016/j.atmosenv.2004.12.047.

20. Kleeman MJ, Schauer JJ, Cass GR. Size and composition distribution of fine particulate matter emitted from motor vehicles. Environ Sci Technol. 2000;34(7):1132-42. doi:10.1021/es981276y.

21. Wahlström J, Olander L, Olofsson U. Size, shape, and elemental composition of airborne wear particles from disc brake materials. Tribol Lett. 2009;38(1):15-24. doi:10.1007/s11249-009-9564-x.

22. Nieuwenhuijsen MJ, Gómez-Perales JE, Colvile RN. Levels of particulate air pollution, its elemental composition, determinants and health effects in metro systems. Atmos Environ. 2007;41(37):7995-8006. doi:10.1016/j. atmosenv.2007.08.002.

\section{Submit your manuscript to a SpringerOpen ${ }^{\odot}$ journal and benefit from:}

- Convenient online submission

- Rigorous peer review

- Immediate publication on acceptance

- Open access: articles freely available online

- High visibility within the field

- Retaining the copyright to your article

Submit your next manuscript at $>$ springeropen.com 